\title{
Does the Utilization of Allograft Tissue in Medial Patellofemoral Ligament Reconstruction in Pediatric and Adolescent Patients Restore Patellar Stability?
}

\author{
Eric Hohn MD, Nirav K. Pandya MD
}

Published online: 2 September 2016

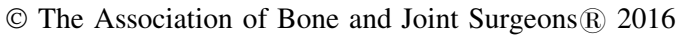

\begin{abstract}
Background Medial patellofemoral ligament (MPFL) reconstruction is one of several surgical procedures used to treat patellofemoral instability. Use of allograft tissue can preserve autogenous tissue and may be preferable in patients with connective tissue disorders or ligamentous laxity. Although there are successful reports in adults, it is unclear if the use of allograft tissue in MPFL reconstruction can restore patellofemoral stability in children and adolescents.

Questions/purposes (1) Does allograft tissue in MPFL reconstruction in pediatric and adolescent patients restore patellar stability? (2) What complications were associated
\end{abstract}

One of the authors certifies that he (NKP) has received payments during the study period of less than USD 10,000 from Orthopediatrics (Warsaw, IN, USA).

All ICMJE Conflict of Interest Forms for authors and Clinical Orthopaedics and Related Research ${ }^{\mathbb{R}}$ editors and board members are on file with the publication and can be viewed on request.

Clinical Orthopaedics and Related Research ${ }^{\circledR}$ neither advocates nor endorses the use of any treatment, drug, or device. Readers are encouraged to always seek additional information, including FDAapproval status, of any drug or device prior to clinical use. Each author certifies that his institution approved the human protocol for this investigation and that all investigations were conducted in conformity with ethical principles of research.

This work was performed at UCSF Benioff Children's Hospital Oakland, Oakland, CA, USA.

E. Hohn

San Francisco Orthopaedic Residency Program, San Francisco, CA, USA

N. K. Pandya ( $\square)$

Department of Orthopaedic Surgery, University of California

San Francisco, UCSF Benioff Children's Hospital Oakland, 747

52nd Street, Oakland, CA 94605, USA

e-mail: Nirav.Pandya@ucsf.edu with allograft MPFL reconstructions in children and adolescents?

Methods Between June 2012 and August 2015, one surgeon (NKP) performed $26 \mathrm{MPFL}$ reconstructions in 23 patients with gracilis allograft for traumatic patellar instability. Of those, 25 (96\%) were available for followup more than 1 year later (mean, 24 months; range, 12-44 months). During this time, the surgeon suggested reconstruction to patients who had recurrent dislocation or subluxation after 6 weeks of bracing, physical therapy, and activity modification if they were noted to have a torn or attenuated MPFL on MRI. During that period, this was the only surgical technique the surgeon used to treat traumatic patellar instability. Patients undergoing concurrent bony procedures were ineligible for inclusion. The mean age of the patients in the series was $16.0( \pm 2)$ years. Age, sex, skeletal maturity, presence of trochlear dysplasia, and additional arthroscopic procedures at the time of reconstruction were collected. Postoperative notes and imaging were reviewed for presence of complications defined as recurrent dislocation, recurrent subluxations, fractures, infection, or arthrofibrosis. These complications were identified by chart review by the senior surgeon (NKP) and study personnel (EH) not involved in clinical care of the patients or by patient-reported complications. Recurrent subluxation or dislocation was patient-reported at the time of the clinic visit or followup phone/email contact. Fractures were defined as any cortical disruption in the femur or patella that required treatment (change in postoperative protocol), infection requiring treatment (antibiotics and/or return to the operating room), or arthrofibrosis (stiffness that necessitated a change in the postoperative protocol or manipulation under anesthesia).

Results Ninety-two percent (23 of 25) of patients reported no further instability episodes after MPFL reconstruction. 
Sixteen percent (four of 25) of patients had complications: two repeat episodes of patellar instability, one patella fracture, and one symptomatic hardware requiring interference screw removal. No patients developed arthrofibrosis or infection.

Conclusions In this small case series, we found that MPFL reconstruction using allograft tissue in children and adolescents resulted in a low risk of recurrent instability, perhaps comparable to what has been published by others who have used autograft tissue. Longer followup is needed, because in some orthopaedic applications, allograft ligaments have been observed to attenuate over time. Future studies might compare these techniques using patient-reported outcomes scores as well as use a control group of patients with autograft tissue.

Level of Evidence Level IV, therapeutic study.

\section{Introduction}

Patellar dislocations are among the most common knee injuries in the pediatric population $[10,16]$. Unlike the adult population, the risk of recurrent instability is high [18]. Although the treatment of patients with first-time patellar dislocations remains controversial [39], recurrent dislocations in this population may benefit from surgical intervention. The medial patellofemoral ligament (MPFL) is the major medial soft tissue restraint that prevents lateral displacement of the patella during the first $30^{\circ}$ of flexion [2] and is commonly injured after dislocation [3, 35]. Although primary repair of the MPFL has been reported to prevent recurrent patellar instability [43], there are also data to suggest a high risk of recurrent instability with this approach [22, 29]. MPFL reconstruction has been described as a successful treatment for preventing recurrent patellar instability in children and adolescents $[11,17,21,25-28,31,32,46]$. Redislocation is uncommon, occurring in between $0 \%$ and $20 \%$ of patients [21, 25, 32]. However, Parikh et al. [32] noted complications in $16.2 \%$ of patients including recurrent instability, patellar fractures, patellofemoral pain, and loss of flexion. Multiple techniques have been described for MPFL reconstruction in children and adolescents with both autograft and allograft tissue [1, 5, 8, 11, 12, 17, 21, 25-28, 30-33, 37, 40-42, 46]. Allograft tissue has potential advantages of limiting donor site morbidity including loss of strength, decreased surgical time, and use in patients with tissue disorders $[4,13,20,23,24]$, yet it is important to note that these studies did not look at donor site morbidity from use of a single hamstring tendon (as opposed to a double hamstring harvest), which is used in MPFL reconstructions that theoretically may be less pronounced. In adults, allograft tissue has shown comparable results in regard to preventing recurrent patellar instability when used for MPFL reconstruction as compared with autograft $[8,45]$. However, we are aware of only one report in children about allograft MPFL reconstructions [19]. Allograft reconstruction of the anterior cruciate ligament in active children and adolescents is associated with a higher risk of failure when compared with autograft $[6,9,15]$, but it is unknown whether this risk applies to allograft MPFL reconstruction, because the forces this ligament experiences are different from those seen by the anterior cruciate ligament.

We therefore asked: (1) Does allograft tissue in MPFL reconstruction in pediatric and adolescent patients restore patellar stability? (2) What complications were associated with allograft MPFL reconstructions in children and adolescents?

\section{Patients and Methods}

Between June 2012 and August 2015 one surgeon (NKP) performed $26 \mathrm{MPFL}$ reconstructions with gracilis allograft for patients with traumatic patellar instability. Of those, 25 $(96 \%)$ were available for followup more than 1 year later (mean, 24 months; range, 12-44 months). During this time, the surgeon suggested reconstruction to patients who had recurrent dislocation or subluxation after 6 weeks of bracing, physical therapy, and activity modification, if they were noted to have a torn or attenuated MPFL on MRI. During that period, this was the only surgical technique the surgeon used to treat traumatic patellar instability. Patients were ineligible for inclusion if they had concomitant bony procedures including tibial tubercle transfers, osteotomies (for rotation or angular deformity), guided growth procedures, metabolic bone disease, neuromuscular disorders, or connective tissue disease. A minimum of 1 year of followup was required. In addition, they were excluded if they did not have preoperative MRI. Institutional review board approval was obtained for this retrospective study.

Twenty-three patients (three patients underwent bilateral surgery) met the initial inclusion criteria for the study representing 26 surgeries. One patient was lost to followup before the minimum of 1 year and so therefore was not included in the final analysis for a total of 25 patients. Patients who did not return for a scheduled visit after their 1-year followup were contacted by email or phone. All patients had some manner of followup within the last 2 years. Demographic data including age at time of surgery, sex, medical and surgical history, and affected extremity were collected. Preoperative radiographs and MRI were also evaluated in all patients. Skeletal maturity was assessed to determine if the physes were open or closed. MRIs were used to evaluate for the presence of trochlear 
dysplasia. Intraoperative images and operative notes were reviewed as well.

The mean age of patients was $16.0( \pm 2)$ years with seven males and 18 females. Mean followup was $24( \pm 10)$ months. No patients had a history or examination consistent with ligamentous laxity, 16 patients had closed physes at the time of surgery, and 21 patients presented with trochlear dysplasia. Fourteen patients required additional arthroscopic procedures at the time of MPFL reconstruction including chondroplasty of the femoral condyle or patella (nine), arthroscopic lateral release (five), loose body removal (three), patellar microfracture (one), partial lateral meniscectomy (one), and partial medial meniscectomy (one).

\section{Surgical Technique}

All patients first underwent a standard diagnostic arthroscopy to evaluate patellar tracking as well as additional intraarticular pathology. This was followed by open MPFL reconstruction. Using a medial parapatellar incision, the superomedial edge of the patella was exposed.

Under fluoroscopic guidance, taking care not to violate the articular surface of the patella, guide pins were placed across the patella, parallel to the joint line. The first was placed $3 \mathrm{~mm}$ distal to the superomedial corner of the patella and a second placed 10 to $15 \mathrm{~mm}$ distal and parallel to the first (Fig. 1). The guide pins were then overdrilled with a cannulated reamer taking care not to violate the far cortex. The thawed gracilis allograft was whipstitched 10 $\mathrm{mm}$ at each end using \#2 braided nonabsorbable suture and secured into the patellar drill holes both proximally and distally with interference fixation. All grafts were obtained from Allosource (Centennial, CO, USA) and were frozen. The grafts were irradiated at a very low dose at the end of packaging to achieve the desired sterility level.

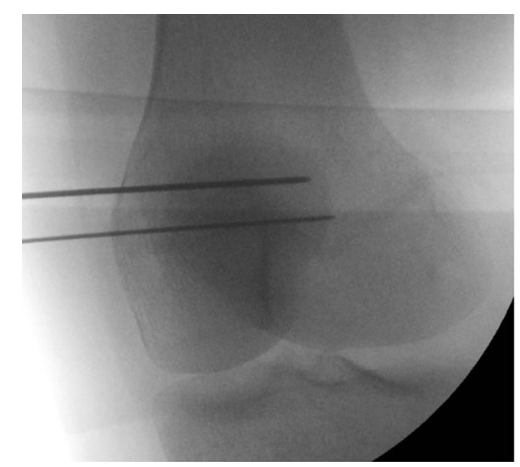

Fig. 1 AP fluoroscopic image demonstrating patellar guide pin placement. The first was placed $3 \mathrm{~mm}$ distal to the superomedial corner of the patella and a second placed 10 to $15 \mathrm{~mm}$ distal and parallel to the first.
The femoral insertion site was then identified under fluoroscopic control as described by Schöttl et al. [34], and a guide pin was placed percutaneously at this site (Fig. 2). The guide pin was advanced across the femur bicortically and its position was confirmed radiographically making sure it did not cross the distal femoral physis. A 2- to 3-cm skin incision around the guide pin was made, and the guide pin was then overreamed with a reamer to the far cortex of the femur. In patients who were skeletally immature, both femoral guide pin placement and reaming were done under fluoroscopic guidance to ensure there was no physeal violation. Blunt dissection was then used to develop the interval between the capsule and the vastus medialis and the graft was passed from the patellar incision to the femoral incision. The graft was then passed into the femoral tunnel with the use of a passing suture around the looped gracilis graft, and the passing suture ends were delivered out the lateral skin. The graft was tensioned with the lateral patella flush with the lateral femoral condyle with the knee flexed at $30^{\circ}$, taking care to have equal tension on both graft ends. The isometry was then tested by cycling the knee through ROM. The graft was then fixed in place with a bioabsorbable screw (Fig. 3). Standard wound closure was performed and patients were discharged home with crutches and a ROM brace.

\section{Rehabilitation}

All patients underwent a standard rehabilitation protocol. This included toe-touch weightbearing with crutches and a hinged knee brace locked in extension for 6 weeks postoperatively. Physical therapy was initiated 1 week postoperatively to begin knee ROM in the hinged knee brace. Knee ROM began at $0^{\circ}$ to $30^{\circ}$ and advanced to $0^{\circ}$ to $90^{\circ}$ by 6 weeks postoperatively. At 6 weeks postoperatively, the crutches and knee brace were discontinued. At 4 months after surgery patients were allowed to run and sporting activity was allowed at 6 months if patients demonstrated clinical stability. A graduated physical therapy program was used to allow return to sports. Patients were on average followed at 6-week intervals until 6

Fig. 2 Lateral fluoroscopic imaging demonstrating the femoral guide pin insertion site. 


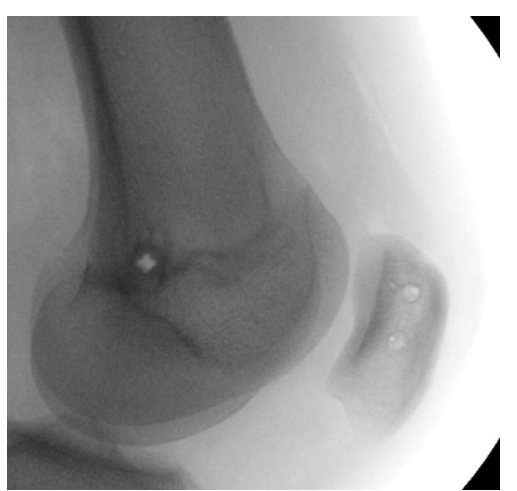

Fig. 3 Final lateral fluoroscopic imaging demonstrating fixation in the patella and femur after the graft has been secured.

months after surgery and then 6 months after full return to sport.

\section{Assessment of Endpoints}

Postoperative notes and imaging were reviewed for presence of complications defined as recurrent dislocation, recurrent subluxations, fractures, infection, or arthrofibrosis. These complications were identified by chart review by the senior surgeon (NKP) and study personnel (EH) not involved in clinical care of the patients or through patientreported complications by phone/email. Recurrent subluxation or dislocation was patient-reported at the time of the clinic visit and/or follow phone/email. Fractures were defined as any cortical disruption in the femur or patella that required treatment (change in postoperative protocol), infection requiring treatment (antibiotics and/or return to the operating room), or arthrofibrosis (stiffness that necessitated a change in the postoperative protocol or manipulation under anesthesia).

\section{Results}

Ninety-two percent (23 of 25) of patients reported no instability episodes at latest followup and had returned to their prior activity levels. Two patients sustained repeat dislocations, one who had trochlear dysplasia. No patients had recurrent subluxations.

Four of 25 patients (16\%) developed postoperative complications. Two patients sustained repeat dislocations mentioned previously, one patient sustained a patella fracture as a result of a fall 6 months after surgery, and one patient underwent reoperation 17 months after surgery to remove a biointerference screw on the femoral side that was causing her irritation. No patients had an infection or developed arthrofibrosis.

\section{Discussion}

MPFL reconstruction is commonly used to treat patellofemoral instability both in children and adults. Allograft tissue can be used for ligament reconstruction, and although it has been noted to be used successfully in adults $[8,45]$, it is unclear if the use of allograft tissue in MPFL reconstruction will work as well in children. This of particular concern given the high risk of failure of allograft tissue in anterior cruciate ligament reconstruction in this population $[6,9,15]$. In a cohort of children and adolescents, we found that allograft MPFL reconstruction was associated with only an $8 \%$ rate of recurrent instability.

This study has a number of limitations. First, this was a relatively small sample size of patients without a control group of patients who underwent surgery with autograft tissue for comparison. Therefore, we cannot be certain whether this approach would be better or inferior to other approaches. Our study can be considered preliminary and may be used to help sample size calculations for future comparative trials between allograft MPFL reconstruction and other approaches; we believe such studies will be important in answering the questions posed in this article. Second, we did not collect patient-reported outcome scores. This is an important limitation, because without validated outcomes tools, our assessment is limited to the relatively basic endpoints of recurrent instability and major complications. Future studies on this topic should gather patientreported outcome scores, because those will help us to compare this approach with other available options in a more nuanced manner, particularly as it relates to the preservation of autogenous tissue and postoperative pain and function. Future studies should also look at the specific risk factors for recurrent instability and will require multicenter efforts. Third, because allograft tissue can attenuate over time [7, 14], longer term followup of our patients will be important to ensure that these reconstructions remain durable in this population of young patients even given our mean followup of 2 years. Fourth, during this time period, not all patients underwent reconstruction for instability, which does represent a degree of patient selection bias, yet our indications were reasonable and consistent; the senior author (NKP) suggested reconstruction to patients who had recurrent dislocation or subluxation after 6 weeks of bracing, physical therapy, and activity modification, if they were noted to have a torn or attenuated MPFL on MRI. During that period, this was the only surgical technique the surgeon used to treat traumatic patellar instability. Finally, the risk of growth disturbance in performing the procedure is important for the clinician to consider. We had only nine patients with open physes at the time of the procedure with a mean age of the patients of $14( \pm 2)$ years. Therefore, although these patients are skeletally immature, they represent a group that 
was nearing skeletal maturity. We also did not obtain longstanding films unless an abnormality was noted clinically as a result of the advanced age of the patients. No patients developed any clinically significant growth abnormalities (ie, leg length discrepancies or angular deformities). We do agree that a larger study with a much younger population of patients could address the risk of growth disturbance using this technique in a more skeletally immature population.

We found a low risk of repeat instability using allograft gracilis for MPFL reconstruction in children and adolescents with traumatic patellar instability, we believe comparable to other studies about allograft MPFL reconstructions in adults. In adults, Calvo Rodríguez et al. [8] compared 13 knees that underwent MPFL reconstruction with hamstring autograft versus 16 knees with allograft and found no recurrent dislocations in either group. Weinberger et al. [45] performed a systematic review comparing autograft and allograft and found no difference between either graft choice (recurrence rate of $5.7 \%$ for autograft and $6.7 \%$ for allograft) [45]. The observed risk of recurrence in our study seems similar to those of those other studies, which have examined allograft tissue in the adult population. Our results likewise seem similar to MPFL reconstruction with autogenous tissue in children and adolescents. Lind et al. [21] reported a $20 \%$ redislocation rate in their series of 20 children between the ages of 8 and 16 years with the utilization of autogenous tissue. Parikh et al. [32] in a series of 179 knees found a redislocation rate of $4.6 \%$, and Nelitz et al. [25] in their series of 21 knees had a $9.5 \%$ rate of continued apprehension after reconstruction with autogenous tissue. Even in the context of trochlear dysplasia (present in 21 of our 25 knees), which places additional strain on the MPFL as a result of lack of bony constraint, our recurrence rates were low. Only one of our patients with a recurrence had trochlear dysplasia. Other authors have reported a correlation between worse outcomes and degree of trochlear dysplasia after MPFL reconstruction [36, 44]. However, because allograft tissue can attenuate over time [7, 14], longer term followup of our patients will be important to ensure that these reconstructions remain durable in this population of young patients.

The use of allograft tissue in our study resulted in an overall complication rate of $16 \%$, which seems similar to what has been reported for both autogenous and allograft tissue in adults as well as autograft tissue in the pediatric and adolescent populations. Calvo Rodríguez et al. [8] noted complications in $19 \%$ of adults treated with allograft tissue, including one revision procedure resulting from poor anchor placement, one patellar fracture, and one patient who developed postoperative arthrofibrosis. Singhal et al. [38] examined nine different studies representing 320 MPFL reconstructions done with hamstring autograft and found a complication rate of $12.5 \%$, including cases of arthrofibrosis and patellar fracture. In the pediatric and adolescent populations, Parikh et al. [32] noted an overall complication rate of $16.2 \%$ including eight cases of arthrofibrosis, six patellar fractures, and five cases of prolonged pain. As a result, taking overall complication rate into consideration, allograft tissue utilization in our study was similar to what has been reported for autograft and allograft in adults as well as autograft in pediatric patients. It is important to note that all allografts are not equivalent and that preparation differences may make historical allograft literature less applicable than the historical autograft literature. It is also important to note that rehabilitation protocols differed not only between these studies, but also with the protocol used at our institution. This can be a factor that accounts for the difference in the incidence of complications between the different papers.

In this small case series, we found that MPFL reconstruction using allograft tissue in children and adolescents resulted in a low risk of recurrent instability, perhaps comparable to what has been published by others who have used autograft tissue. Longer followup is needed, because in some orthopaedic applications, allograft ligaments have been observed to attenuate over time [7, 14]. Future studies will be performed with a control group of patients using autogenous tissue with patient-reported outcomes to further understand graft choice in the pediatric and adolescent populations.

\section{References}

1. Ahmad CS, Brown GD, Stein BS. The docking technique for medial patellofemoral ligament reconstruction: surgical technique and clinical outcome. Am J Sports Med. 2009;37:20212027.

2. Amis AA, Firer P, Mountney J, Senavongse W, Thomas NP. Anatomy and biomechanics of the medial patellofemoral ligament. Knee. 2003;10:215-220.

3. Balcarek P, Walde TA, Frosch S, Schüttrumpf JP, Wachowski MM, Stürmer KM, Frosch KH. Patellar dislocations in children, adolescents and adults: a comparative MRI study of medial patellofemoral ligament injury patterns and trochlear groove anatomy. Eur J Radiol. 2011;79:415-420.

4. Barrera Oro F, Sikka RS, Wolters B, Graver R, Boyd JL, Nelson B, Swiontkowski MF. Autograft versus allograft: an economic cost comparison of anterior cruciate ligament reconstruction. Arthroscopy. 2011;27:1219-1225.

5. Bitar AC, Demange MK, D'Elia CO, Camanho GL. Traumatic patellar dislocation: nonoperative treatment compared with MPFL reconstruction using patellar tendon. Am J Sports Med. 2012;40:114-122.

6. Bottoni CR, Smith EL, Shaha J, Shaha SS, Raybin SG, Tokish JM, Rowles DJ. Autograft versus allograft anterior cruciate ligament reconstruction: a prospective, randomized clinical study with a minimum 10-year follow-up. Am J Sports Med. 2015;43:2501-2509.

7. Bullis DW, Paulos LE. Reconstruction of the posterior cruciate ligament with allograft. Clin Sports Med. 1994;13:581-597. 
8. Calvo Rodríguez R, Figueroa Poblete D, Anastasiadis Le Roy Z, Etchegaray Bascur F, Vaisman Burucker A, Calvo Mena R. Reconstruction of the medial patellofemoral ligament: evaluation of the clinical results of autografts versus allografts. Rev Esp Cir Ortop Traumatol. 2015;59:348-353.

9. Engelman GH, Carry PM, Hitt KG, Polousky JD, Vidal AF. Comparison of allograft versus autograft anterior cruciate ligament reconstruction graft survival in an active adolescent cohort. Am J Sports Med. 2014;42:2311-2318.

10. Fithian DC. Epidemiology and natural history of acute patellar dislocation. Am J Sports Med. 2004;32:1114-1121.

11. Giordano M, Falciglia F, Aulisa AG, Guzzanti V. Patellar dislocation in skeletally immature patients: semitendinosous and gracilis augmentation for combined medial patellofemoral and medial patellotibial ligament reconstruction. Knee Surg Sports Traumatol Arthrosc. 2012;20:1594-1598.

12. Gomes JE. Comparison between a static and a dynamic technique for medial patellofemoral ligament reconstruction. Arthroscopy. 2008;24:430-435.

13. Greis PE, Koch BS, Adams B. Tibialis anterior or posterior allograft anterior cruciate ligament reconstruction versus hamstring autograft reconstruction: an economic analysis in a hospital-based outpatient setting. Arthroscopy. 2012;28:16951701.

14. Jackson DW, Corsetti J, Simon TM. Biologic incorporation of allograft anterior cruciate ligament replacements. Clin Orthop Relat Res. 1996;324:126-133.

15. Kaeding CC, Pedroza AD, Reinke EK, Huston LJ; MOON Consortium, Spindler KP. Risk factors and predictors of subsequent ACL injury in either knee after ACL reconstruction: prospective analysis of 2488 primary ACL reconstructions from the MOON cohort. Am J Sports Med. 2015;43:1583-1590.

16. Kraus T, Švehlík M, Singer G, Schalamon J, Zwick E, Linhart W. The epidemiology of knee injuries in children and adolescents. Arch. Orthop Trauma Surg. 2012;132:773-779.

17. Kumahashi N, Kuwata S, Tadenuma T, Kadowaki M, Uchio Y. A 'sandwich' method of reconstruction of the medial patellofemoral ligament using a titanium interference screw for patellar instability in skeletally immature patients. Arch Orthop Trauma Surg. 2012;132:1077-1083.

18. Lewallen LW, McIntosh AL, Dahm DL. Predictors of recurrent instability after acute patellofemoral dislocation in pediatric and adolescent patients. Am J Sports Med. 2013;41:575-581.

19. Li G, Sun XB, Ni JT, Cao L, Zhang KY. [Treatment of instability of patellofemoral joint through arthroscopic repair of medial patellofemoral ligament for adolescents with epiphyseal non-closure by allogenic tendon] [in Chinese]. Zhonghua $Y i$ Хие Za Zhi. 2011;91:2468-2471.

20. Li S, Su W, Zhao J, Xu Y, Bo Z, Ding X, Wei Q. A metaanalysis of hamstring autografts versus bone-patellar tendon-bone autografts for reconstruction of the anterior cruciate ligament. Knee. 2011;18:287-293.

21. Lind M, Enderlein D, Nielsen T, Christiansen SE, Faun $\varnothing$ P. Clinical outcome after reconstruction of the medial patellofemoral ligament in paediatric patients with recurrent patella instability. Knee Surg Sports Traumatol Arthrosc. 2016;24:666671.

22. Matic GT, Magnussen RA, Kolovich GP, Flanigan DC. Return to activity after medial patellofemoral ligament repair or reconstruction. Arthroscopy. 2014;30:1018-1025.

23. Mohtadi NG, Chan DS, Dainty KN, Whelan DB. Patellar tendon versus hamstring tendon autograft for anterior cruciate ligament rupture in adults. Cochrane Database Syst Rev. 2011;7:CD005960.

24. Nakamura N, Horibe S, Sasaki S, Kitaguchi T, Tagami M, Mitsuoka T, Toritsuka Y, Hamada M, Shino K. Evaluation of active knee flexion and hamstring strength after anterior cruciate ligament reconstruction using hamstring tendons. Arthroscopy. 2002;18:598-602.

25. Nelitz M, Dreyhaupt J, Reichel H, Woelfle J, Lippacher S. Anatomic reconstruction of the medial patellofemoral ligament in children and adolescents with open growth plates: surgical technique and clinical outcome. Am J Sports Med. 2013;41:5863.

26. Nelitz M, Reichel H, Dornacher D, Lippacher S. Anatomical reconstruction of the medial patellofemoral ligament in children with open growth-plates. Arch Orthop Trauma Surg. 2012;132:1647-1651.

27. Nelitz M, Theile M, Dornacher D, Wölfle J, Reichel H, Lippacher S. Analysis of failed surgery for patellar instability in children with open growth plates. Knee Surg Sports Traumatol Arthrosc. 2012;20:822-828.

28. Nelitz M, Williams SR. Anatomic reconstruction of the medial patellofemoral ligament in children and adolescents using a pedicled quadriceps tendon graft. Arthrosc Tech. 2014;3:e303308.

29. Nietosvaara Y, Paukku R, Palmu S, Donell ST. Acute patellar dislocation in children and adolescents. Surgical technique. $J$ Bone Joint Surg Am. 2009;91(Suppl 2):139-145.

30. Nomura E, Inoue M. Hybrid medial patellofemoral ligament reconstruction using the semitendinous tendon for recurrent patellar dislocation: minimum 3 years' follow-up. Arthroscopy. 2006;22:787-793.

31. Nwachukwu BU, So C, Schairer WW, Green DW, Dodwell ER. Surgical versus conservative management of acute patellar dislocation in children and adolescents: a systematic review. Knee Surg Sports Traumatol Arthrosc. 2016;24:760-767.

32. Parikh SN, Nathan ST, Wall EJ, Eismann EA. Complications of medial patellofemoral ligament reconstruction in young patients. Am J Sports Med. 2013;41:1030-1038.

33. Schöttle PB, Fucentes SF, Romero J. Clinical and radiological outcome of medial patellofemoral ligament reconstruction with a semitendinosus autograft for patella instability. Knee Surg Sports Traumatol Arthrosc. 2005;13:516-521.

34. Schöttl PB, Schmeling A, Rosenstiel N, Weiler A. Radiographic landmarks for femoral tunnel placement in medial patellofemoral ligament reconstruction. Am J Sports Med. 2007;35:801-804.

35. Seeley M, Bowman KF, Walsh C, Sabb BJ, Vanderhave KL. Magnetic resonance imaging of acute patellar dislocation in children: patterns of injury and risk factors for recurrence. $J$ Pediatr Orthop. 2012;32:145-155.

36. Shah JN, Howard JS, Flanigan DC, Brophy RH, Carey JL, Lattermann C. A systematic review of complications and failures associated with medial patellofemoral ligament reconstruction for recurrent patellar dislocation. Am J Sports Med. 2012;40:19161923.

37. Sillanpää $P$, Mattila VM, Visuri T, Mäenpää H, Pihlajamäki H. Ligament reconstruction versus distal realignment for patellar dislocation. Clin Orthop Relat Res. 2008;466:1475-1484.

38. Singhal R, Rogers S, Charalambous CP. Double-bundle medial patellofemoral ligament reconstruction with hamstring tendon autograft and mediolateral patellar tunnel fixation: a meta-analysis of outcomes and complications. Bone Joint J. 2013;95:900905.

39. Smith TO, Donell S, Song F, Hing CB. Surgical versus nonsurgical interventions for treating patellar dislocation. Cochrane Database Syst Rev. 2015;2:CD008106.

40. Steensen RN, Dopirak RM, Maurus PB. A simple technique for reconstruction of the medial patellofemoral ligament using a quadriceps tendon graft. Arthroscopy. 2005;21:365-370.

41. Steiner TM, Torga-Spak R, Teitge RA. Medial patellofemoral ligament reconstruction in patients with lateral patellar instability and trochlear dysplasia. Am J Sports Med. 2006;34:1254-1261. 
42. Tang H, Xu YQ, Zheng TE, Sha Y, Xu XS, Zhao WQ, Cui Y, Zhang XJ, Pu SQ, Li Chuan, Li CX. [Anatomical double bundle reconstruction of medial patellofemoral ligament with allograft tendon in the treatment of patellar dislocations] [in Chinese]. Zhongguo Gu Shang. 2015;28:252-255.

43. Tompkins M, Kuenze CM, Diduch DR, Miller MD, Milewski $\mathrm{MD}$, Hart JP. Clinical and functional outcomes following primary repair versus reconstruction of the medial patellofemoral ligament for recurrent patellar instability. J Sports Med (Hindawi Publ Corp). 2014;2014:702358.

44. Wagner D, Pfalzer F, Hingelbaum S, Huth J, Mauch F, Bauer G. The influence of risk factors on clinical outcomes following anatomical medial patellofemoral ligament (MPFL) reconstruction using the gracilis tendon. Knee Surg Sports Traumatol Arthrosc. 2013;21:318-324.

45. Weinberger JM, Fabricant PD, Taylor SA, Mei JY, Jones KJ. Influence of graft source and configuration on revision rate and patient-reported outcomes after MPFL reconstruction: a systematic review and meta-analysis. Knee Surg Sports Traumatol Arthrosc. 2016 Feb 8. [Epub ahead of print]

46. Yercan HS, Erkan S, Okcu G, Ozalp RT. A novel technique for reconstruction of the medial patellofemoral ligament in skeletally immature patients. Arch Orthop Trauma Surg. 2011;131:1059-1065. 